\title{
Kit-Build Concept Mapping for Being Aware of the Gap of Exchanged Information in Collaborative Reading of the Literature
}

\author{
Yusuke Hayashi and Tsukasa Hirashima \\ The Department of Information, Hiroshima University, Japan \\ \{hayashi, tsukasa\}@lel.hiroshima-u.ac.jp
}

\begin{abstract}
This paper reports a result of the trial use of the kit-build method in a lesson conducted in a graduate school. The purpose of the lesson is that students understand the content of a book collaboratively. The problem to be addressed here is that it is difficult to acknowledge the discrepancies between a presentation and their own understanding. To solve it this study uses Kit-build concept mapping. Through collaborative reading of the literature with kit-build concept mapping in classroom students could find the problems in their own presentation and tried to refine it with concrete information about the others' misunderstanding.
\end{abstract}

Keywords: E-learning and e-education, concept map, collaborative knowledge building.

\section{Introduction}

Collaborative knowledge building activity deepens understanding of the participants of the activity [2]. For example, in constructive collaborative problem solving activity, participants are required to explain their own understanding to the others and to compare it with ones of the others. These activities help them to grasp their own understanding and induce reflection on it. This is collaborative knowledge building activity and contributes to making understanding deeper [3]. Consequently, they build knowledge to solve the problem collaboratively.

Here, it is considered that one of the most important factor for successful is to share information related to the problem. If they couldn't share information about the problem, they wouldn't have a basis for thinking to solve the problem. This study aims at improving the quality of information exchange for collaborative knowledge building. As the first step of that, this study investigates the effectiveness of a method to build concept map against being aware of the gap between information of a provider and receivers in exchange of it.

This study uses a special method to build concept map called Kit-build concept mapping [4]. In this method concept maps cannot be built freely. An information provider makes a concept map as the correct answer. This is called goal map. And then, this is decomposed into separated nodes and likes. These parts are provides to 
receivers and they make a concept map with the parts to represent their understanding. This method doesn't allow freedom to build concept maps. Instead, an information provider can grade maps of receivers with consistency as the degree of the same parts as the gal map. The degree can be an important indicator of the gap between understanding of a provider and receivers.

This paper report a result of the trial use of the kit-build method in a lesson conducted in a graduate school. The purpose of the lesson is that students understand the content of a book collaboratively. Each student reads a part of the book and provides the contents of the part to the others. Through this activity students can obtain information of the contents of the whole of the book.

This lesson adopts the jigsaw method [1] for making groups of students in order to that learners divide task to read a book and understand the content collaboratively. The characteristics of this method are to make students develop a feeling of responsibility and to facilitate them to examine a relationship between the contents. In this lesson each student is assigned to a chapter and has a feeling of responsibility to explain it to the others. Only after they finish to explain their parts each other they get information about the whole contents of the book.

When students explain the contents their assigned chapter, students play two roles of provider and receiver by turns. For example, there are three students, LA, LB and LC. In the first turn LA is the provider and the rests are the receivers. In the following turns LB and LC become the providers and the rests in each turn become the receivers respectively. All the students become provider more than one time in this manner. In this lesson each student plays provider twice.

The purpose of these activities is to let the students be aware of the gap between provider's understanding and receiver's one. After a provider makes a presentation to the others in his or her group, receivers make a concept map with a kit of a map. In the first turn, this is a task for receivers to organize information that they are provided and also is a task for provider to be aware of the gap between what he or she want to tell to the others and what the others understand from his or her presentation. This is a collaborative knowledge building activity for the contents of the books. And then, by the second turn the provider improve his or her presentation with other students assigned the same chapter. They exchange information of problems happened in their explanation about their assigned chapter and discuss solution about it. This is another collaborative knowledge building activity for way of presentation. Finally, the provider presents the same contents with modification again in the second turn and judge the modification by himself or herself.

As the result, some students are aware of the gap between what he or she explains and what the others receive. And based on that they try to improve their presentations or goal maps.

\section{Kit-Build Concept Mapping in Collaborative Reading}

The main purpose of this study is to investigate the effectiveness of kit-build concept map system in collaborative reading of the literature in classroom. The supposed 
lesson style is that students read an assigned part of the literature and make a presentation to explain it. This is a typical lesson in Japanese university. Through this process students share the content of the literature.

This study aims at enables students be aware of the gap between information providers and receivers. When a person tells something for other people, it is not true that they receive it as it is. There could be discrepancies between them. The goal of this lesson is to share the content of the literature. It is preferred that the presenter perceives the state of achievement of the audience and gives feedback for correcting misunderstanding of them effectively. The problem is that it is difficult to acknowledge the discrepancies in a presentation.

This study adopts two methods to conduct effective collaborative reading of the literature in classroom. One is the jigsaw method that is a cooperative learning technique in which learners are divided into two types of groups, jigsaw and expert, and learn through collaboration between the types of groups. The other is kit-build concept mapping that is a special method to build concept maps from a kit provided by a teacher.

The system for kit-build mapping is called "KBmap system". This is composed of two client systems: "KBmap editor" and "KBmap analyzer", and the server system "KBmap DB" [5].

There are two types of KBmap editor that work on desktop and tablet computers. The KBmap editor on desktop computers is for both teachers and learners. Teachers can make goal-map and kit for learners and learners can make their map from a kit on the desktop version. On the other hand, tablet version is only for learners. Figure 1 shows KBeditor on tablet computers and Figure 2 shows the screenshot. The left side shows a kit that includes separated nodes and links. The right side is a KBmap made from the kit. The characteristic of the tablet version is portability. It can be used in not computer rooms but normal classrooms. In this study lessons were done in a normal classroom with Wi-Fi network.

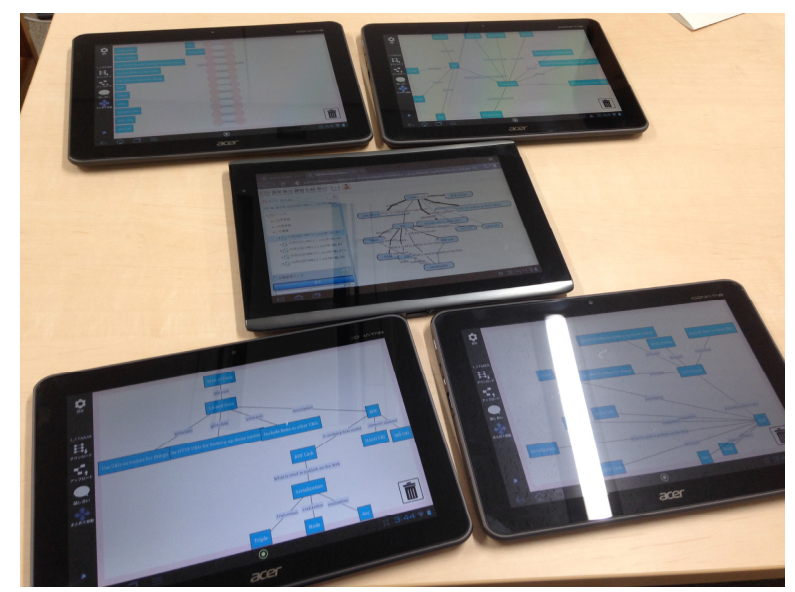

Fig. 1. KBeditor on tablet computers 
KBmap analyzer work on only web browsers and can work on tablet computers. In this study students used KBanalyzer on tablet computers in the classroom in order to analyze KBmaps made by other students. The characteristic of KBanalyzer is to show the group map that that overlays learner-maps. On this map the more learner set a link between particular concepts, the thicker the link is displayed. Figure 3 shows a screenshot of KBanalyzer. On the window links many learners set are displayed as thick and high-colored lines and links few learners set are displayed as narrow and light colored lines. In addition to that, KBanalyzer shows each link made by learners. With this information a teacher can identify which links in goal-map are difficult for learners to set when they make their maps from a kit [6].

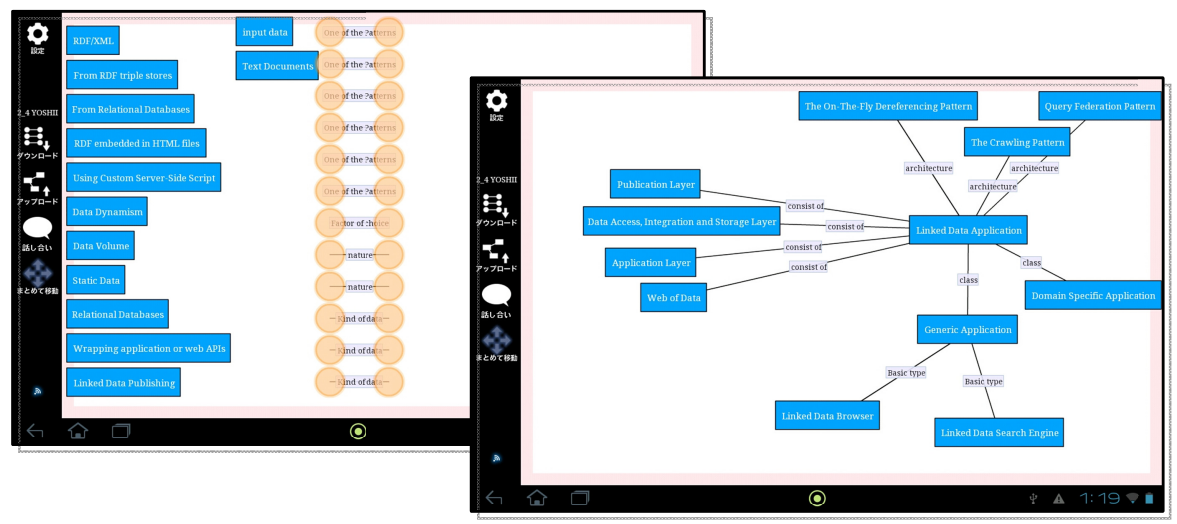

Fig. 2. The screenshot of KBeditor on tablet computers

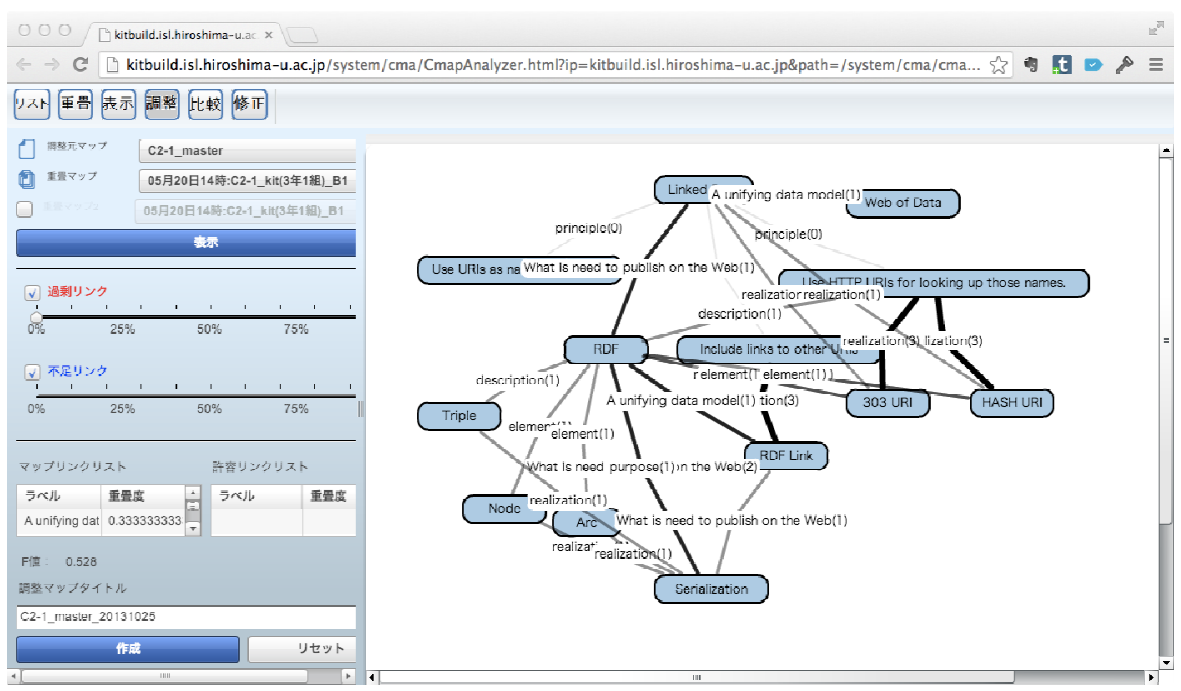

Fig. 3. A screenshot of KBanalyzer 


\section{Collaborative Reading of the Literature with Kit-Build Concept Mapping in Classroom}

This course has two purposes; to understand the subject domain and to polish up presentation skills of students. To achieve them, This study adopts jigsaw method. This is a cooperative learning technique in which learners are divided into two types of groups, jigsaw and expert, and learn through collaboration between the types of groups. In a jigsaw group, learners learn the same topic together. This is the preparation for the group work in an expert group. The goal of the jigsaw group is that each learner understands the topic well because they join different expert group separately. An expert group is composed of members from different jigsaw group. This means that an expert group is a group of experts of different topics. In the group learners teach their expert topic each other and understand the whole topics.

In this study jigsaw group is for learning the content of the same assigned chapter. On the other hand, expert group is composed of learners from jigsaw group and cover the whole book. The book used in this curriculum is composed of seven chapters. Five of them except the first and the last chapter are used in jigsaw group. The participants are 41 graduate school students whose major is computer science. They are divided into jigsaw groups of five or six people and expert group of four or five people. Figure 4 shows the composition of jigsaw and expert groups. This class had ten jigsaw groups and eight expert groups. The expert groups can be classified into two clusters because two jigsaw groups are made for each chapter. Each student organizes the content of the assigned chapter and then teaches it to the other members in the expert group. In addition to that, the results in the expert groups are gathered and used for improvement of presentation in the jigsaw group.

The lesson structure is the following:

\section{Introduction}

2. First turn of presentation

3. Second turn of presentation

Firstly, as the introduction to this lesson, the author gave a lecture about the subject domain, which is the linked data in this case and an instruction of kit-build concept map system. The instruction included two parts that were about kit-build concept mapping (KB-mapping) and the analysis of kit-build maps (KB-analysis). The instruction followed the lecture. The author asked the students to make a concept map (learner-map) by a kit made from the concept map the author made (goal-map). After that, as the instruction of $\mathrm{KB}$-analysis, the author showed the result of KB-mapping on kit-build map analyzer system (KB-analyzer). KB-analyzer can show not only the result and the correctness of KB-mapping of each learner but also group-map that overlays learner-maps.

The rest of the curriculum is the turn of the learners. Learners had exchanges of the content of the book assigned to them. In this lesson each learner makes presentations twice. They organize the content of assigned chapter and make a goal-map and a 
presentation. The goal-map becomes the criteria to evaluate the others' understanding of a presentation as well as a guideline to make the presentation. In the first and second turns, learners give presentation to the other group members and check their understanding by KB mapping with the goal-map each other. In addition to that each learner reflects on his or her presentation with the result of KB-mapping and then improve his or her presentation for the second turn.

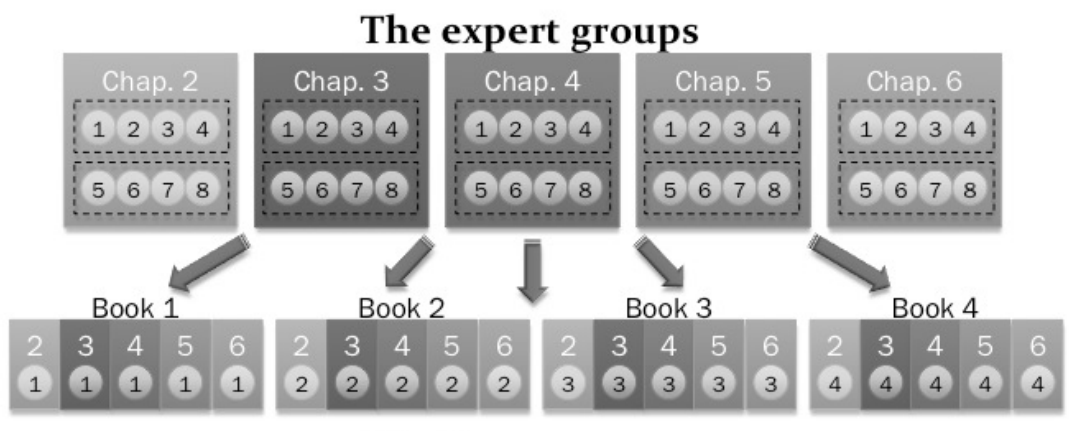

\section{The jigsaw groups}

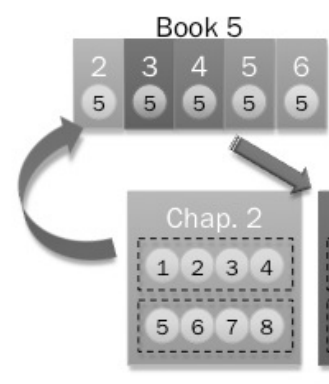

Book 6

Book 7

Book 8

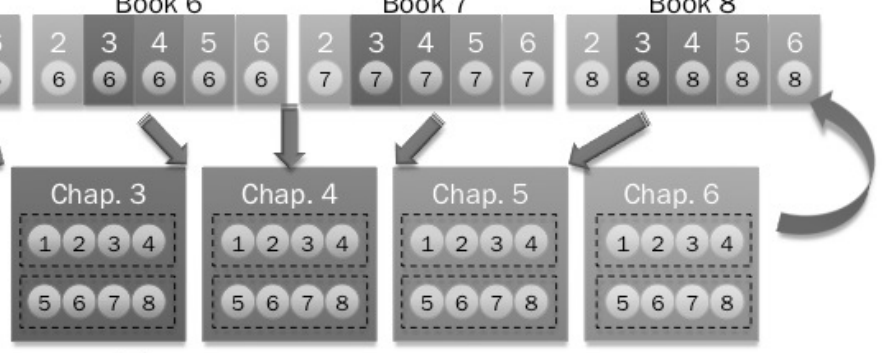

The expert groups

Fig. 4. The composition of jigsaw and expert groups

\section{The Results and Consideration}

Table 1 shows the change of map score of students. For each chapter from second to sixth each excepting one he or she made a presentation to the others, students basically made two KBmaps in each of the first and second turns. However, in the first turn of the second chapter, all the students made only one KBmap. It is because that it takes much time for students to be familiar with this style of lesson so that there was no time to make KBmap again. In the second turn of the sixth chapter some students also made KBmap only once. There is another reason for that. In this case the students were able to make the correct KBmap on the first attempt so that there was no need to make KBmap again.

From Figure 5 (A1) to (B2) Illustrate the change of map-score of student chapter by chapter. These data is classified by goal map and turn of presentation. For each 
Table 1. The scores in the first turn

\begin{tabular}{|c|c|c|c|c|c|c|c|c|c|c|c|}
\hline & \multicolumn{10}{|c|}{ Chapter and 1st/2nd mapping } \\
\hline & & C2-1 & C2-2 & C3-1 & C3-2 & C4-1 & C4-2 & C5-1 & $\mathrm{C} 5-2$ & C6-1 & C6-2 \\
\hline \multirow{10}{*}{ 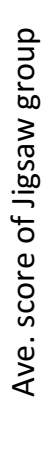 } & 1 & 0.40 & - & 0.13 & 1.00 & 0.26 & 0.81 & 0.63 & 1.00 & 0.79 & 0.91 \\
\hline & 2 & 0.28 & - & 0.78 & 0.94 & 0.37 & 0.85 & 0.56 & 0.71 & 0.50 & 1.00 \\
\hline & 3 & 0.23 & - & 0.75 & 1.00 & 0.44 & 0.85 & 0.81 & 1.00 & 0.64 & 1.00 \\
\hline & 4 & 0.35 & - & 0.38 & 0.82 & 0.29 & 0.97 & 0.79 & 0.96 & 0.87 & 0.89 \\
\hline & $1-4$ & 0.31 & & 0.51 & 0.94 & 0.34 & 0.87 & 0.70 & 0.92 & 0.70 & 0.95 \\
\hline & 5 & 0.24 & - & 0.57 & 0.81 & 0.31 & 0.87 & 0.93 & 1.00 & 0.95 & 0.93 \\
\hline & 6 & 0.41 & - & 0.57 & 0.81 & 0.41 & 0.95 & 1.00 & 1.00 & 0.95 & 0.95 \\
\hline & 7 & 0.14 & - & 0.67 & 0.97 & 0.53 & 1.00 & 0.70 & 0.88 & 0.95 & 0.95 \\
\hline & 8 & 0.25 & - & 0.50 & - & 0.33 & 0.51 & 0.82 & 0.93 & 0.86 & 1.00 \\
\hline & $5-8$ & 0.26 & & 0.58 & 0.86 & 0.39 & 0.83 & 0.86 & 0.95 & 0.93 & 0.96 \\
\hline
\end{tabular}

Table 2. The scores in the second turn

\begin{tabular}{|c|c|c|c|c|c|c|c|c|c|c|c|}
\hline & \multicolumn{10}{|c|}{ Chapter and 1st/2nd mapping } \\
\hline & & C2-1 & $\mathrm{C} 2-2$ & C3-1 & $\mathrm{C} 3-2$ & C4-1 & $\mathrm{C} 4-2$ & C5-1 & C5-2 & C6-1 & C6-2 \\
\hline \multirow{10}{*}{ 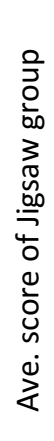 } & 1 & 0.58 & 0.97 & 0.15 & 0.97 & 0.71 & 0.79 & 0.94 & 1.00 & 0.93 & 1.00 \\
\hline & 2 & 0.75 & 0.79 & 0.55 & 1.00 & 0.81 & 1.00 & 0.77 & 1.00 & 0.91 & 1.00 \\
\hline & 3 & 0.88 & 1.00 & 0.45 & 1.00 & 0.78 & 0.88 & 0.81 & 1.00 & 0.93 & 1.00 \\
\hline & 4 & 0.83 & 1.00 & 0.24 & 0.98 & 0.73 & 1.00 & 0.93 & 1.00 & 0.89 & 0.95 \\
\hline & $1-4$ & 0.76 & 0.94 & 0.35 & 0.99 & 0.76 & 0.92 & 0.87 & 1.00 & 0.92 & 0.99 \\
\hline & 5 & 0.69 & 0.75 & 0.62 & 1.00 & 0.90 & 1.00 & 0.94 & 1.00 & 1.00 & - \\
\hline & 6 & 0.63 & 0.81 & 0.65 & 0.87 & 0.68 & 1.00 & 0.79 & 0.97 & 1.00 & - \\
\hline & 7 & 0.70 & 0.78 & 0.82 & 0.96 & 0.58 & 1.00 & 0.88 & 0.93 & 1.00 & - \\
\hline & 8 & 0.56 & 0.85 & 0.96 & 1.00 & 0.70 & 0.88 & 0.97 & 1.00 & 0.86 & 0.80 \\
\hline & $5-8$ & 0.67 & 0.83 & 0.68 & 0.96 & 0.72 & 0.96 & 0.89 & 0.98 & 0.95 & 0.89 \\
\hline
\end{tabular}

chapter two jigsaw group were made in this course. And more, as mentioned above, each student made KBmaps in two turns. The second turn was done five weeks later from the first turn.

These graphs shows similar tendency in the two jigsaw group categories. From Fig. 5 (A1) and (B1), in the first turn, the score in the first map-making increased with progression of the course. There might be some reasons for this phenomenon. The students could be familiar with the topic and the style of lessons and learn from others' presentation and feedbacks. It is difficult to distinguish and identify the reason from the data obtained in this study. On the other hand, the score in the second map 
making was not changed through the chapters. This is just the effect of feedback. The presenter gave feedback to the other members based on the information on KBanalyzer. However, the style of feedback was not regulated completely so that some students almost directly told correct answers to the other members.

(A1) jigsaw group 1-4, 1st turn, 1st-2nd mapping

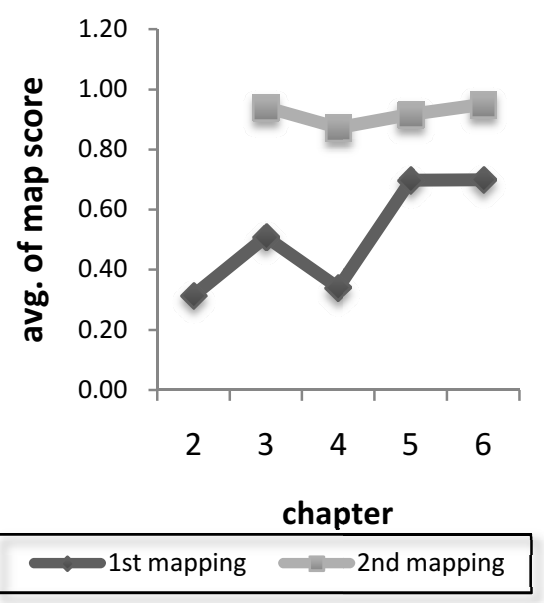

(B1) jigsaw group 5-8, 1st turn, 1st-2nd mapping

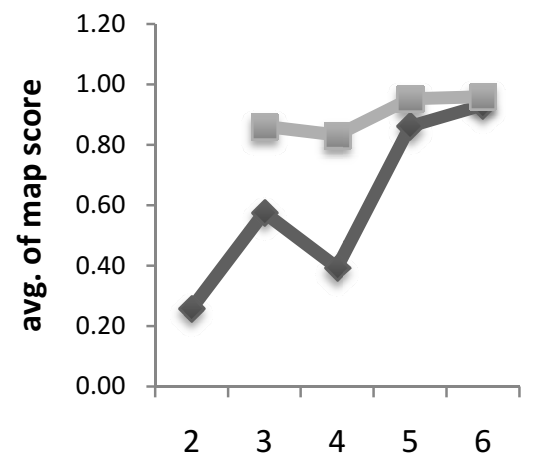

chapter

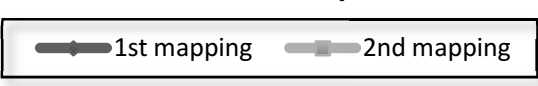

(A1) jigsaw group 1-4, 2nd turn, 1st-2nd mapping

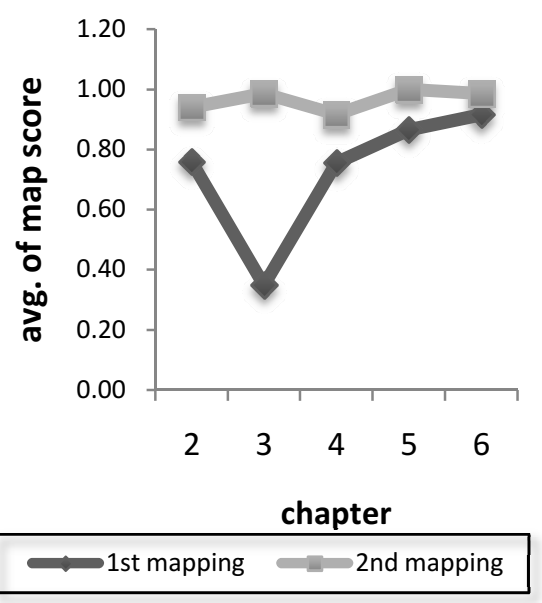

(B2) jigsaw group 5-8, 2nd turn, 1st-2nd mapping

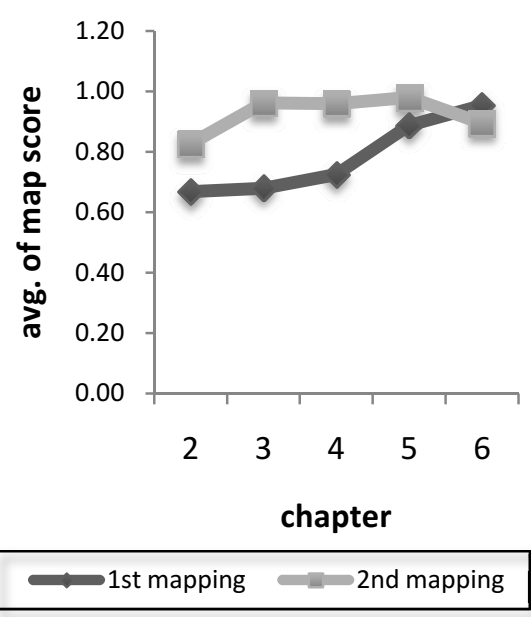

Fig. 5. Changes of scores of each jigsaw group categories 
From Figure 5 (A2) and (B2), in the second turn, the score even in the first map making was not changed through chapters. Especially, from the second to fourth chapter, the scores are basically increased from the first turn. This could be the effect of reflection on the first presentation with KB analyzer. Students were allowed to use KBanalyzer between the first and second presentations. In addition to that, the author gave a task to students to make a report that described which information on KBanalyzer affected to improve the second presentation. The improvement could be always successful. At the third chapter in Figure 5 (A2) the score is decreased from the first turn. Although the students changed presentation to improve, this led the negative effect. However, the group succeeded to increase the score as long as the other chapters.

Finally, Figure 6 summarizes the change of score of students. In both categories of jigsaw groups score is increased from the first turn to the scone turn. All the increase are significant as shown in Table 3.

Table 3. Change of score in jigsaw group

\begin{tabular}{|c|c|c|c|c|c|}
\hline & \multicolumn{3}{|c|}{ Ave. score of Jigsaw group } \\
\hline & & & $1^{\text {st }}$ turn & $2^{\text {nd }}$ turn & Diff. $1^{\text {st }}-2^{\text {nd }}$ turn \\
\hline \multirow{6}{*}{ 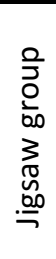 } & & $1^{\text {st }}$ mapping & 0.51 & 0.92 & $0.41(p<.001)$ \\
\hline & $1-4$ & $2^{\text {nd }}$ mapping & 0.73 & 0.97 & $0.24(p<.05)$ \\
\hline & & Diff. $1^{\text {st }}-2^{\text {nd }}$ mapping & 0.22 & 0.05 & \\
\hline & & $1^{\text {st }}$ mapping & 0.60 & 0.90 & $0.30(p<.001)$ \\
\hline & $5-8$ & $2^{\text {nd }}$ mapping & 0.78 & 0.92 & $0.14(p=.073)$ \\
\hline & & Diff. $1^{\text {st }}-2^{\text {nd }}$ mapping & 0.18 & 0.02 & \\
\hline
\end{tabular}

(C1) jigsaw group 1-4, 1st-2nd turn, 1st-2nd mapping

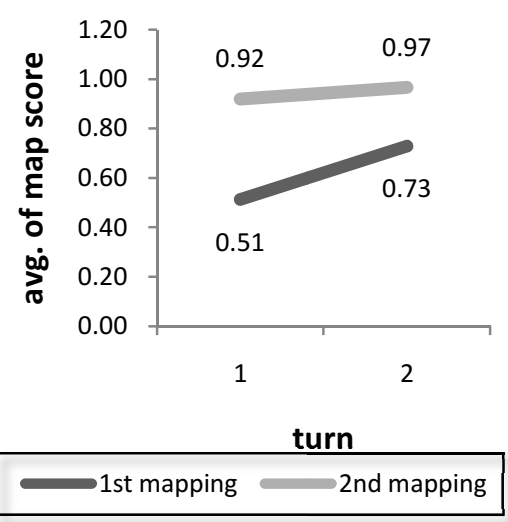

(C2) jigsaw group 5-8, 1st-2nd turn, 1st-2nd mapping

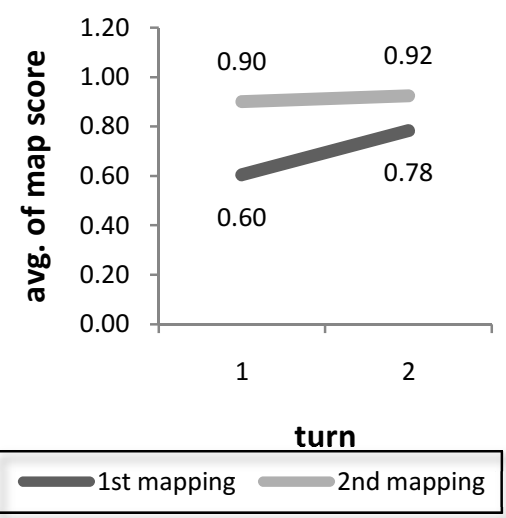

Fig. 6. Change of score in jigsaw group 


\section{Conclusion}

This paper reports the effectiveness of kit-build concept map system in collaborative reading of the literature in classroom. The purpose of the lesson is that students understand the content of a book collaboratively. In such class it is difficult for students as audiences to acknowledge the discrepancies between a presentation and their own understanding.

To solve it this study uses Kit-build concept mapping. Through collaborative reading of the literature with kit-build concept mapping in classroom students could find the problems in their own presentation and tried to refine it with concrete information about the others' misunderstanding.

The result shows in each turn students as presenters could find which part is difficult for the others to understand and give feedback to them. In addition to that, presenters could refine their presentation based on the result. Of course, the increase of the score might mainly come from the presentation is the second time. On the other hand some students, in their reports, stated that they had improved their presentation based on the result of the first presentation and had been confident of success in the second presentation. This shows there could be a possibility of KB concept mapping to be aware of the discrepancies between a presentation and understanding of the audience. It is necessary to identify the scope and the extent of the effect of this style of lesson and improve it further more in the future.

\section{References}

1. Aronson, E., Blanney, N., Sephan, C.: The jigsaw classroom, Beverly Hills. Sage Publications, CA (1978)

2. Bransford, J.D., Brown, A.L., Cocking, R.R.: How people learn: Brain, mind, experience, and school. National Academies Press, Washington, DC (1999)

3. Chi, M.T.H.: Self-explaning: The dual processes, of generating interface and repairing mental models. In: Glaser, R. (ed.) Advances in Instructional Psychology, Educational Design and Cognitive Science, vol. 5, pp. 161-238. Lawrence Erlbaum, Mahwah (2000)

4. Hirashima, T., Yamasaki, K., Fukuda, H., Funaoi, H.: Kit-build concept map for automatic diagnosis. In: Biswas, G., Bull, S., Kay, J., Mitrovic, A. (eds.) AIED 2011. LNCS, vol. 6738, pp. 466-468. Springer, Heidelberg (2011)

5. Sugihara, K., Osada, T., Nakata, S., Funaoi, H., Hirashima, T.: Experimental Evaluation of Kit-Build Concept Map for Science Classes in an Elementary School. In: Proc. of ICCE 2012, Main Conference E-Book, pp. 17-24 (2012)

6. Yoshida, K., Sugihara, K., Nino, Y., Shida, M., Hirashima, T.: Practical Use of Kit-Build Comcept Map System for Formative Assessment of Learners' Comprehension in a Lecture. In: Proc of ICCE 2013, pp. 892-901 (2013) 